\title{
Dual-threshold symbol selective relaying in cooperative networks
}

\author{
Peiyao Yang, Hai Li, and Shujuan Hou* \\ School of Information and Electronics, Beijing Institute of Technology, Beijing 100081, China
}

\begin{abstract}
In order to efficiently mitigate error propagation and reduce computational complexity, this paper proposes a scheme for traditional cooperative networks, named as dual-threshold symbol selective demodulate-and-forward. In the scheme, two log likelihood ratio (LLR)-based thresholds are devised to measure the reliability of received signals for the relay and the destination, respectively. One of the threshold guarantees that the relay only forwards reliable symbols, thus less error will be propagated to the destination. The other threshold is used at the destination for determining the reliability of symbols received from the source. The destination will directly demodulate reliable symbols received from the source. Otherwise, when the symbols received from the source are not reliable, the maximum ratio combiner (MRC) is used to combine symbols received from the source and the relay. Closed-form expression of the bit error probability (BEP) of the proposed scheme is derived and analyzed under binary phase shift keying (BPSK) modulation. Then, the relationship and closed-form solutions of two LLR-based thresholds are derived. Simulation results prove that the theoretical BEP of the proposed scheme closely matches the simulated ones. The proposed scheme can achieve high performance with low computational complexity compared to existing schemes.
\end{abstract}

Keywords: demodulate-and-forward, relaying strategies, error propagation, cooperative networks.

DOI: $10.21629 / J S E E .2017 .06 .03$

\section{Introduction}

Cooperative relaying strategies can be classified into two categories [1]: (i) amplify-and-forward (AF), where the signals are amplified by the relay and then forwarded to the destination, and (ii) decode-and-forward (DF), where the signals being decoded and remodulated before forwards to the destination. For AF, the destination needs full knowledge of the channel-state-information (CSI) of each link. The scheme becomes complicated when the CSI is unknown. For DF, there is no error propagation compared with AF because DF relaying only forwards symbols upon

\footnotetext{
Manuscript received December 16, 2016

*Corresponding author.

This work was supported by the National High Technology Research and Development Program of China (863 Program) (2014AA2649).
}

successful decoding. However, there will be few frames being forwarded to the destination when the signal-tonoise ratio (SNR) between the source and the relay is too low. In that case, the relay cannot successfully decode most received signals [2].

In order to reduce the computation complexity in DF at the relay, demodulate-and-forward (DmF) was proposed in [3]. The relay demodulates the received signals and then forwards remodulated symbols to the destination without decoding. However, signals that are forwarded by the relay have equal weights and do not carry any information about the degree of uncertainty [4,5]. Thus, DmF can be viewed as a suboptimal scheme and cannot effectively mitigate error propagation.

To mitigate error propagation in DmF, threshold-based DmF relaying schemes were introduced, including SNRbased selective relaying schemes and LLR-based selective relaying schemes [6-21]. For SNR-based schemes [616], the received signals at the relay will be detected and forwarded to the destination only when their SNR is above a predefined threshold. However, SNR-based schemes rely on that the relay knows the instantaneous SNR of the source-destination (S-D) link. Once the instantaneous SNR cannot be obtained accurately, the applying of the average SNR will degrade the system performance [6]. For log likelihood ratio (LLR)-based schemes [17-21], the authors derive the theoretical BEP and a near-optimal threshold is calculated by minimizing the system bit error probability (BEP).

The above studies only focus on the error propagation mitigation on the relay side. To further improve the BEP performance, Ikki et al. [22] demonstrated that the error performance highly depends on the threshold employed at the destination. Zeng et al. [23,24] proposed joint threshold-based relaying and maximum-likelihood (ML) detection at the destination, and the optimal threshold of the relay is obtained by minimizing the BEP using the exhaustive grid search method. However, performance analysis of ML detection is quite complicated especially for 
general constellations, and the grid search method requires a large number of function evaluations in practical application.

In order to solve this problem, some studies have focused on designing the receiver at the destination, including $\lambda$-maximum ratio combiner (MRC) [25] and C-MRC [26,27]. However, for $\lambda$-MRC, since the combiner parameter $\lambda$ has not been specified analytically, fulldiversity claims cannot be proved [26]. For C-MRC, the instantaneous BEP of the S-R link is required at the destination.

Motivated by the previous research, we aim at exploiting the LLR-based thresholds at the relay and at the destination in this paper, respectively. In the proposed scheme, the relay detects and forwards symbols whose magnitude of LLR is greater than a predetermined threshold to mitigate error propagation. An LLR-based threshold is also used at the destination to measure the reliability of received symbols from the S-D link. In order to analyze the performance and obtain the thresholds of the proposed scheme, the close-form BEP expression is derived in this paper. Different from the previous studies, closed-form solutions of the thresholds are derived in this paper.

The rest of this paper is organized as follows. In Section 2, we introduce the notation and the system model. In Section 3, the closed-form expression of BEP and the expressions of thresholds are derived. In Section 4, we provide simulation results that validate the theoretical analysis of our proposal. Finally, conclusions are given in Section 5 .

\section{System model}

In this paper, a traditional two-hop relay system is used, including a source node $\mathrm{S}$, a relay node $\mathrm{R}$ and a destination node D, as shown in Fig. 1.

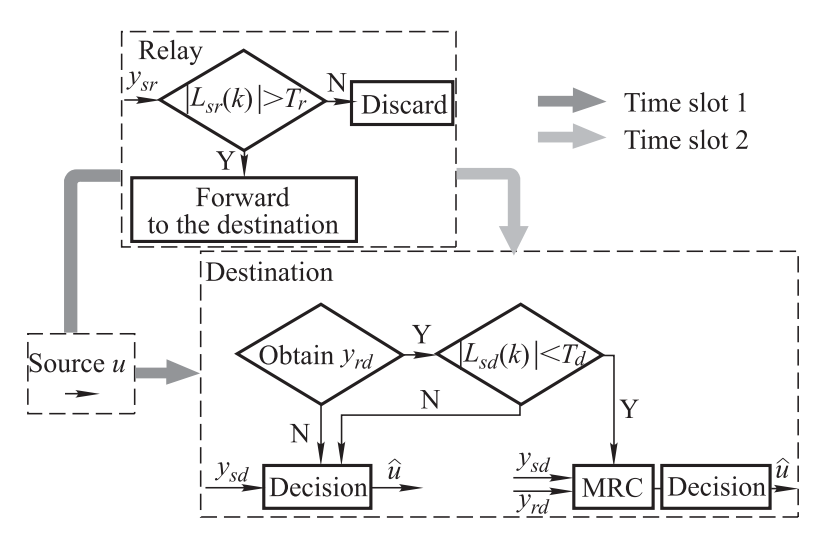

Fig. 1 System model

In Fig. 1, transmission is divided into time slot 1 and time slot 2 . In time slot 1 , the source transmits symbols to both the relay and the destination. The signals received at the relay and at the destination are expressed as

$$
\begin{aligned}
& r_{s r}=h_{s r} u_{s}+w_{s r} \\
& r_{s d}=h_{s d} u_{s}+w_{s d}
\end{aligned}
$$

where $u_{s} \in\{ \pm 1\}$ is the symbol transmitted by the source under BPSK modulation with equal probability. $w_{s r}$ and $w_{s d}$ are complex additive white Gaussian noise (AWGN) signals with variance $\sigma_{s r}^{2}$ and $\sigma_{s d}^{2}$, respectively. $h_{s r}$ and $h_{s d}$ represent the Rayleigh channel fading coefficients of the S-R link and S-D link, respectively. The channel fading coefficients are defined as

$$
\begin{aligned}
& E\left[\left|h_{s r}\right|^{2}\right]=\left(d_{s r}\right)^{-\gamma} \\
& E\left[\left|h_{s d}\right|^{2}\right]=\left(d_{s d}\right)^{-\gamma}
\end{aligned}
$$

where $d_{i j}$ is the $i$ - $j$ link distance, $\gamma$ is the path-loss exponent.

The received signal after channel compensation for S-R link can be expressed as

$$
y_{s r}=\operatorname{Re}\left\{h_{s r}^{*} r_{s r}\right\}=\left|h_{s r}\right|^{2} u_{s}+\left|h_{s r}\right|\left|w_{s r}\right| .
$$

In time slot 2 , the hard-decision of received symbol while its LLR is greater than a preset threshold $T_{r}$ is forwarded by the relay. Otherwise, the relay will keep silent. The signal received from the relay to destination (R-D) link is expressed as

$$
r_{r d}=h_{r d} u_{r}+w_{r d}
$$

where $u_{r} \in\{ \pm 1\}$ is the remodulated symbol at the relay under BPSK.

At the destination, an LLR threshold $T_{d}$ is applied to distinguish reliable symbols from unreliable symbols of the S-D link signals $y_{s d}$. If the relay forwards the symbol to the destination, and the LLR of $y_{s d}$ is less than $T_{d}$, which we call unreliable symbol, the destination will combine both the S-D link signal $y_{s d}$ and the R-D link signal $y_{r d}$ by MRC [28] in the time slot 2. Otherwise, the destination will perform the detection only based on the received signal from the S-D link. The LLR of received symbol from S-D link can be calculated by

$$
\begin{gathered}
L_{s d}=\ln \frac{P\left(y_{s d} \mid h_{s d}, x_{s}=+1\right)}{P\left(y_{s d} \mid h_{s d}, x_{s}=-1\right)}= \\
\frac{2}{\sigma_{s d}^{2}} y_{s d}=L_{c}^{s d} y_{s d}
\end{gathered}
$$

where

$$
L_{c}^{s d}=\frac{2}{\sigma_{s d}^{2}} .
$$

The received signal at the destination after MRC can be expressed as

$$
y_{M R C}=L_{s d}+L_{r d}, \quad\left|L_{s d}\right| \leqslant T_{d},\left|L_{s r}\right|>T .
$$




\section{Performance analysis}

\subsection{Computation complexity analysis}

In the proposed scheme, MRC is used at the destination when the relay forwards symbols to the destination and the symbols received from the source are not reliable. Since an LLR-based threshold is applied at the destination, fewer symbols from the source and the relay need to be combined at the destination. This is more efficient than the scheme [23] that requires an ML detector for all symbols from the source and the relay.

The ML detector [23] for BPSK when $\left|L_{s r}\right|>T_{r}$ can be expressed as

$$
\hat{x}_{d}^{M L} \approx \arg \max _{\alpha \in\{ \pm 1\}}\left(\max _{\beta \in\{ \pm 1\}} F_{\alpha, \beta}\right)
$$

where

$$
\begin{gathered}
F_{\alpha, \beta}=\operatorname{Re}\left\{\frac{1}{\sigma_{s d}^{2}} h_{s d}^{*} r_{s d} \alpha+\frac{1}{\sigma_{r d}^{2}} h_{r d}^{*} r_{r d} \beta\right\}+ \\
\ln P\left(x_{r}=\beta \mid x_{s}=\alpha\right) .
\end{gathered}
$$

Clearly, comparing (9) with (10), the ML detector is more complex, especially for higher-order constellations. Table 1 presents the computational cost of the proposed method and the scheme [23] at the destination when the relay forwards received reliable symbols.

Table 1 Computation complexity analysis of the proposed method and the scheme [23] at the destination when the relay forwards symbols

\begin{tabular}{ccc}
\hline Method & \multicolumn{2}{c}{ Computational cost } \\
\cline { 2 - 3 } & Multiplication & Addition \\
\hline $\begin{array}{c}\text { Proposed scheme when } \\
\left|L_{s d}\right| \leqslant T_{d},\left|L_{s r}\right|>T_{r}\end{array}$ & $2 \mathrm{~N}$ & $\mathrm{~N}$ \\
\hline $\begin{array}{c}\text { Proposed scheme when } \\
\left|L_{s d}\right|>T_{d},\left|L_{s r}\right|>T_{r}\end{array}$ & $2 \mathrm{~N}$ & 0 \\
\hline Scheme $[23]$ & $2 \mathrm{~N}$ & $8 \mathrm{~N}$ \\
\hline
\end{tabular}

\subsection{BER analysis}

The closed-form expression of E2E BEP can be expressed as

$$
\begin{gathered}
P_{e}(\varepsilon)=P_{e}\left(\varepsilon_{s d},\left|L_{s d}\right|>T_{d}\right)+ \\
P_{e}\left(\varepsilon_{s d},\left|L_{s d}\right| \leqslant T_{d},\left|L_{s r}\right| \leqslant T_{r}\right)+ \\
P_{e}\left(\varepsilon_{M R C},\left|L_{s d}\right| \leqslant T_{d},\left|L_{s r}\right|>T_{r}\right)
\end{gathered}
$$

where $P_{e}\left(\varepsilon_{s d},\left|L_{s d}\right|>T_{d}\right)$ is the BEP at the destination when the signal received from the source $y_{s d}$ is viewed as reliable symbol. $P_{e}\left(\varepsilon_{s d},\left|L_{s d}\right| \leqslant T_{d},\left|L_{s r}\right| \leqslant T_{r}\right)$ is the BEP at the destination when the received signal $y_{s d}$ is not reliable when the relay keep silent. $P_{e}\left(\varepsilon_{M R C},\left|L_{s d}\right| \leqslant\right.$ $\left.T_{d},\left|L_{s r}\right|>T_{r}\right)$ is the BEP at the destination after combination of the S-D link signals $y_{s d}$ and the R-D link signals $y_{r d}$.

The PDF of received signals for $i$ - $j$ link can be ex- pressed as (as shown in Appendix A of [19])

$$
f_{y_{i j}}\left(y_{i j}\right)=\alpha_{i j} \exp \left(\beta_{i j} x_{i} y_{i j}\right) \exp \left(-\chi_{i j}\left|y_{i j}\right|\right)
$$

where

$$
\begin{gathered}
\alpha_{i j}=1 / \sqrt{d_{i j}^{-\alpha} 2 \sigma_{i j}^{2}\left(1+\bar{\gamma}_{i j}\right)} \\
\beta_{i j}=1 / \sigma_{i j}^{2} \\
\chi_{i j}=\sqrt{\left(1+\bar{\gamma}_{i j}\right) / \bar{\gamma}_{i j}} / \sigma_{i j}^{2} .
\end{gathered}
$$

We set

$$
\begin{gathered}
\varphi_{i j}=\frac{\beta_{i j}-\chi_{i j}}{L_{c}^{i j}}=\frac{-P_{B}\left(\bar{\gamma}_{i j}\right)}{1-2 P_{B}\left(\bar{\gamma}_{i j}\right)} \\
\varphi_{i j}^{C}=\frac{\beta_{i j}+\chi_{i j}}{L_{c}^{i j}}=\frac{1-P_{B}\left(\bar{\gamma}_{i j}\right)}{1-2 P_{B}\left(\bar{\gamma}_{i j}\right)} .
\end{gathered}
$$

To calculate (12), we use the expression of BEP with BPSK modulation [29], that is

$$
P_{B}(\bar{\gamma})=\frac{1}{2}\left(1-\sqrt{\frac{\bar{\gamma}}{1+\bar{\gamma}}}\right)
$$

where $\bar{\gamma}$ is the average SNR.

If the LLR of $y_{s d}$ is greater than the threshold $T_{d}$, the destination will directly demodulate signals received from the S-D link. To simplify the analysis of the proposed scheme, we assume that all symbols transmitted from the source is $+1\left(u_{s}=+1\right)$. The error probability can be derived as

$$
\begin{gathered}
P_{e}\left(\varepsilon_{s d},\left|L_{s d}\right|>T_{d}\right)=\int_{-\infty}^{-T_{d} / L_{c}^{s d}} f_{y_{s d}}\left(y_{s d}\right) \mathrm{d} y_{s d}= \\
P_{B}\left(\bar{\gamma}_{s d}\right) \exp \left(-\varphi_{s d}^{C} T_{d}\right) .
\end{gathered}
$$

Thus, if symbols transmitted from the source is $u_{s}=+1$, an error occurs when $L_{s d}$ is negative.

If the LLR of $y_{s d}$ and $y_{s r}$ are less than the threshold $T_{d}$ and $T_{r}$ respectively, the relay will keep silent and only S-D link signals $y_{s d}$ will be considered when the destination performs detection. The error probability can be derived as

$$
\begin{gathered}
P_{e}\left(\varepsilon_{s d},\left|L_{s d}\right| \leqslant T_{d},\left|L_{s r}\right| \leqslant T_{r}\right)= \\
\int_{-T_{r} / L_{c}^{s r}}^{T_{r} / L_{c}^{s r}} \int_{-T_{d} / L_{c}^{s d}}^{0} f_{y_{s d}}\left(y_{s d}\right) f_{y_{s r}}\left(y_{s r}\right) \mathrm{d} y_{s d} \mathrm{~d} y_{s r}= \\
\left(1-P_{B}\left(\bar{\gamma}_{s r}\right) \mathrm{e}^{-\varphi_{s r}^{C} T_{r}}-\left(1-P_{B}(\bar{\gamma})\right) \mathrm{e}^{\varphi_{s r} T_{r}}\right) . \\
P_{B}\left(\bar{\gamma}_{s d}\right)\left(1-\mathrm{e}^{-\varphi_{s d}^{C} T_{d}}\right) .
\end{gathered}
$$

If the LLR of $y_{s d}$ is less than the threshold $T_{d}$, and the LLR of $y_{s r}$ is greater than the threshold $T_{r}$, the relay will forward the remodulated reliable symbols received from the source to the destination. Then, the destination will combine both the S-D link signals $y_{s d}$ and the R-D link signals $y_{r d}$ by MRC. Thus, the BEP at the destination after MRC can be derived as

$$
P_{e}\left(\varepsilon_{M R C},\left|L_{s d}\right| \leqslant T_{d},\left|L_{s r}\right|>T_{r}\right)=
$$




$$
\begin{gathered}
P_{e}\left(\varepsilon_{\text {prop }}\right) P_{e}\left(\varepsilon_{r},\left|L_{s r}\right|>T_{r}\right)+ \\
P_{e}\left(\varepsilon_{\text {coop }}\right) P\left(\varepsilon_{r}^{C},\left|L_{s r}\right|>T_{r}\right)
\end{gathered}
$$

where $P_{e}\left(\varepsilon_{r},\left|L_{s r}\right|>T_{r}\right)$ is the BEP at the relay when the erroneous detected symbol is selected for retransmission. $P\left(\varepsilon_{r}^{C},\left|L_{s r}\right|>T_{r}\right)$ is the probability at the relay when the symbol is detected as correct. $P_{e}\left(\varepsilon_{\text {prop }}\right)$ is the BEP at the destination when the relay forwards erroneous detected symbol and an MRC combiner is used at the destination. $P_{e}\left(\varepsilon_{\text {coop }}\right)$ is the BEP at the destination when the relay forwards correctly detected symbol and an MRC combiner is used at the destination.

In the proposed relaying scheme, only reliable symbols are forwarded to the destination in order to reduce the influence of error propagation. However, if the relay makes an incorrect detection and the corresponding symbols are selected for retransmission, the error probability at the destination can be derived as

$$
\begin{gathered}
P_{e}\left(\varepsilon_{\text {prop }}\right)=P_{e}\left(\varepsilon_{M R C},\left|L_{s d}\right| \leqslant T_{d}\left|\varepsilon_{r},\right| L_{s r} \mid>T_{r}\right)= \\
\int_{-T_{d} / L_{c}^{s d}}^{T_{d} / L_{c}^{s d}} \int_{-\infty}^{-L_{c}^{s d} y_{s d} / L_{c}^{r d}} f_{y_{s d}}\left(y_{s d}\right) f_{y_{r d}}\left(y_{r d}\right) \mathrm{d} y_{r d} \mathrm{~d} y_{s d}= \\
\frac{\alpha_{s d}}{L_{c}^{s d}} \frac{1-P_{B}\left(\bar{\gamma}_{r d}\right)}{\varphi_{s d}+\varphi_{r d}}\left(\mathrm{e}^{\left(\varphi_{s d}+\varphi_{r d}\right) T_{d}}-1\right)+ \\
\frac{\alpha_{s d}}{L_{c}^{s d}} \frac{-P_{B}\left(\bar{\gamma}_{r d}\right)}{\varphi_{s d}^{C}+\varphi_{r d}^{C}}\left(1-\mathrm{e}^{-\left(\varphi_{s d}^{C}+\varphi_{r d}^{C}\right) T_{d}}\right)+ \\
P_{B}\left(\bar{\gamma}_{s d}\right)\left(1-\mathrm{e}^{-\varphi_{s d}^{C} T_{d}}\right) .
\end{gathered}
$$

When the relay detects symbols correctly, the error event will occur at the destination after the combination of the S-D link signals $y_{s d}$ and the R-D link signals $y_{r d}$. The error probability at the destination can be derived as

$$
\begin{gathered}
P_{e}\left(\varepsilon_{\text {coop }}\right)=P_{e}\left(\varepsilon_{M R C},\left|L_{s d}\right| \leqslant T_{d}\left|\varepsilon_{r}^{C},\right| L_{s r} \mid>T_{r}\right)= \\
\int_{-T_{d} / L_{c}^{s d}}^{T_{d} / L_{c}^{s d}} \int_{-\infty}^{-L_{c}^{s d} y_{s d} / L_{c}^{r d}} f_{y_{s d}}\left(y_{s d}\right) f_{y_{r d}}\left(y_{r d}\right) d y_{r d} d y_{s d}= \\
\frac{\alpha_{s d}}{L_{c}^{s d}} \frac{2 P_{B}\left(\bar{\gamma}_{r d}\right)-1}{\varphi_{s d}-\varphi_{r d}^{C}}\left(\mathrm{e}^{\left(\varphi_{s d}-\varphi_{r d}^{C}\right) T_{d}}-1\right)+ \\
P_{B}\left(\bar{\gamma}_{s d}\right)\left(1-\mathrm{e}^{-\varphi_{s d}^{C} T_{d}}\right) .
\end{gathered}
$$

Substituting the result of (20) and (21) into (19), the BER after MRC is derived. Then the BER of the proposed system is derived by inserting (17), (18) and (19) into (12), the BER expression of the proposed system can be obtained by

$$
\begin{gathered}
P_{e}\left(\varepsilon_{d}\right)=P_{B}\left(\bar{\gamma}_{s d}\right)+ \\
\frac{\alpha_{s d}}{L_{c}^{s d}} \frac{2 P_{B}\left(\bar{\gamma}_{r d}\right)-1}{\varphi_{s d}-\varphi_{r d}^{C}}\left(\mathrm{e}^{\left(\varphi_{s d}-\varphi_{r d}^{C}\right) T_{d}}-1\right)\left(1-P_{B}\left(\bar{\gamma}_{s r}\right)\right) \mathrm{e}^{\varphi_{s r} T_{r}}+
\end{gathered}
$$

$$
\begin{aligned}
& \frac{\alpha_{s d}}{L_{c}^{s d}} \frac{1-P_{B}\left(\bar{\gamma}_{r d}\right)}{\varphi_{s d}+\varphi_{r d}}\left(\mathrm{e}^{\left(\varphi_{s d}+\varphi_{r d}\right) T_{d}}-1\right) P_{B}\left(\bar{\gamma}_{s r}\right) \mathrm{e}^{-\varphi_{s r}^{C} T_{r}}+ \\
& \frac{\alpha_{s d}}{L_{c}^{s d}} \frac{-P_{B}\left(\bar{\gamma}_{r d}\right)}{\varphi_{s d}^{C}+\varphi_{r d}^{C}}\left(1-\mathrm{e}^{-\left(\varphi_{s d}^{C}+\varphi_{r d}^{C}\right) T_{d}}\right) P_{B}\left(\bar{\gamma}_{s r}\right) \mathrm{e}^{-\varphi_{s r}^{C} T_{r}}
\end{aligned}
$$

\subsection{Calculation of two thresholds}

Equation (22) can be expressed as

$$
\begin{gathered}
P_{e}\left(\varepsilon_{d}\right)=\frac{\alpha_{s d}}{L_{c}^{s d}}\left(1-2 P_{B}\left(\bar{\gamma}_{r d}\right)\right)\left(1-2 P_{B}\left(\bar{\gamma}_{s r}\right)\right) f\left(T_{r}, T_{d}\right)+ \\
P_{B}\left(\bar{\gamma}_{s d}\right)
\end{gathered}
$$

where

$$
\begin{gathered}
f\left(T_{r}, T_{d}\right)=-\frac{\varphi_{s r}^{C}}{\varphi_{s d}-\varphi_{r d}^{C}}\left(\mathrm{e}^{\left(\varphi_{s d}-\varphi_{r d}^{C}\right) T_{d}}-1\right) \mathrm{e}^{\varphi_{s r} T_{r}}- \\
\frac{\varphi_{r d}^{C} \varphi_{s r}}{\varphi_{s d}+\varphi_{r d}}\left(\mathrm{e}^{\left(\varphi_{s d}+\varphi_{r d}\right) T_{d}}-1\right) \mathrm{e}^{-\varphi_{s r}^{C} T_{r}}- \\
\frac{\varphi_{r d} \varphi_{s r}}{\varphi_{s d}^{C}+\varphi_{r d}^{C}}\left(1-\mathrm{e}^{-\left(\varphi_{s d}^{C}+\varphi_{r d}^{C}\right) T_{d}}\right) \mathrm{e}^{-\varphi_{s r}^{C} T_{r}}
\end{gathered}
$$

The thresholds $T_{r}$ and $T_{d}$ can be obtained by minimizing $P_{e}\left(\varepsilon_{d}\right)$, which also means minimizing $f\left(T_{r}, T_{d}\right)$. By calculating the partial derivative of function $f\left(T_{r}, T_{d}\right)$ with respect to $T_{d}$ equal to 0 , we can get the relationship between $T_{r}$ and $T_{d}$ as

$$
T_{d} \approx T_{r}+\ln \left(\frac{1-P_{B}\left(\bar{\gamma}_{s r}\right)}{\varphi_{r d}^{C} P_{B}\left(\bar{\gamma}_{s r}\right)}\right) .
$$

As shown in (25), there exists a one-to-one relationship between $T_{r}$ and $T_{d}$. Therefore, we can calculate the threshold $T_{r}$ when $T_{d}$ is given and vice versa.

By calculating the partial derivative of function $f\left(T_{r}, T_{d}\right)$ with respect to $T_{r}$ equal to 0 , we can get the relationship between $T_{r}$ and $T_{d}$ as

$$
\begin{gathered}
\frac{\partial f\left(T_{r}, T_{d}\right)}{\partial T_{r}} \approx \frac{\varphi_{s r} \varphi_{s r}^{C}}{\varphi_{s d}-\varphi_{r d}^{C}} \mathrm{e}^{\varphi_{s r} T_{r}}+ \\
\frac{\varphi_{r d}^{C} \varphi_{s r} \varphi_{s r}^{C}}{\varphi_{s d}+\varphi_{r d}}\left(\mathrm{e}^{\left(\varphi_{s d}+\varphi_{r d}\right) T_{d}}-1\right) \mathrm{e}^{-\varphi_{s r}^{C} T_{r}}
\end{gathered}
$$

where $\mathrm{e}^{\left(\varphi_{s d}-\varphi_{r d}^{C}\right) T_{d}} \approx 0, \mathrm{e}^{-\left(\varphi_{s d}^{C}+\varphi_{r d}^{C}\right) T_{d}} \approx 0$ and $\frac{\varphi_{r d} \varphi_{s r} \varphi_{s r}^{C}}{\varphi_{s d}^{C}+\varphi_{r d}^{C}} \mathrm{e}^{-\varphi_{s r}^{C} T_{r}} \approx 0$ in high SNR.

The threshold $T_{r}$ can be obtained by substituting (25) into (26), and we apply Taylor series to obtain a closedform solution for $T_{r}$ by

$$
\begin{gathered}
\frac{\varphi_{s d}+\varphi_{r d}}{\varphi_{r d}^{C}\left(\varphi_{s d}-\varphi_{r d}^{C}\right)}=\mathrm{e}^{-T_{r}}\left(1-C_{2} \mathrm{e}^{\left(\varphi_{s d}+\varphi_{r d}\right) T_{r}}\right) \\
\ln \frac{\varphi_{s d}+\varphi_{r d}}{\varphi_{r d}^{C}\left(\varphi_{s d}-\varphi_{r d}^{C}\right)}=-T_{r}+\ln \left(1-C_{1}\right)+
\end{gathered}
$$




$$
\ln \left(1+\frac{-C_{1}}{1-C_{1}}\left(\varphi_{s d}+\varphi_{r d}\right) T_{r}\right)
$$

where $C_{1}=\left(\frac{1-P_{B}\left(\bar{\gamma}_{s r}\right)}{\varphi_{r d}^{C} P_{B}\left(\bar{\gamma}_{s r}\right)}\right)^{\left(\varphi_{s d}+\varphi_{r d}\right)}$.

Consequently, we obtain a closed-form solution for $T_{r}$ as

$$
\begin{gathered}
T_{r} \approx \frac{\left(C_{2}-1\right)}{C_{2}^{2}}+\frac{1}{C_{2}^{2}}\left[\left(C_{2}-1\right)^{2}+\right. \\
\left.2 C_{2}^{2} \ln \left(\frac{\varphi_{r d}^{C}\left(\varphi_{s d}-\varphi_{r d}^{C}\right)}{\varphi_{s d}+\varphi_{r d}}\left(1-C_{1}\right)\right)\right]^{\frac{1}{2}}
\end{gathered}
$$

where $C_{2}=-\frac{C_{1}}{1-C_{1}}\left(\varphi_{s d}+\varphi_{r d}\right)$, and the threshold $T_{d}$ can be derived by (29) and (25).

Unlike the scheme proposed in [23], where the threshold of the relay is obtained by minimizing the BEP using the exhaustive grid search [30], closed form solutions for the thresholds are derived in our scheme. This can reduce the computational cost.

\section{Simulation results}

In this section, we verify the proposed scheme by simulations.

Fig. 2 shows the relationship between $T_{r}$ and $T_{d}$. As shown in Fig. 2, the thresholds derived from (29) and (25) match well with the thresholds obtained from simulations. Further, with given $T_{r}$, the analysis threshold $T_{d}$ by (25) accords with the simulation ones too, which proves the linearity of two thresholds in (25).

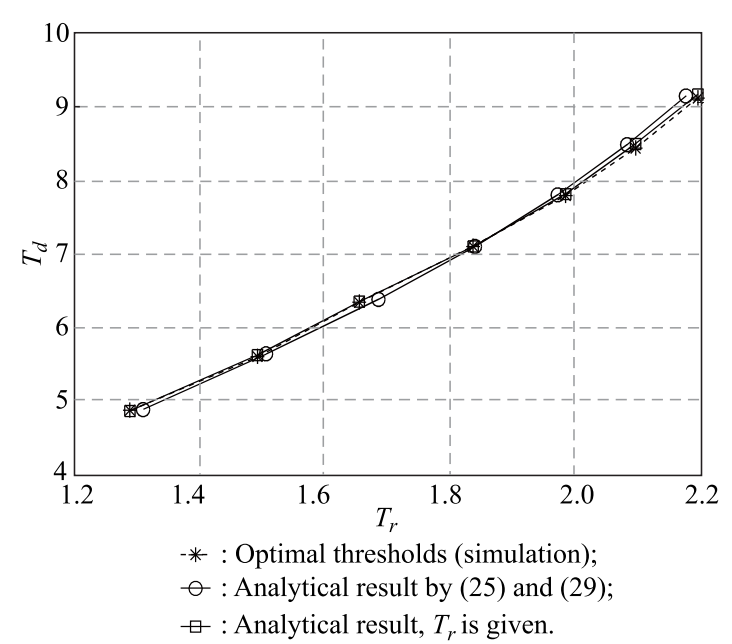

Fig. 2 Theoretical and simulation results of two thresholds $T_{r}$ and $T_{d}$ with different SNR when $d_{s d}=1, d_{s r}=d_{r_{d}}=1 / 2$, $\bar{\gamma}_{s d}=\bar{\gamma}_{s r}=\bar{\gamma}_{r d}$

Based on the relationship between $T_{r}$ and $T_{d}$ in (25), we can plot BEP as a function of $T_{r}$ in Fig. 3. It shows that the simulation result matches well with the theoretical ones. The BEP firstly decreases and then increases when $T_{r}$ increases. This can be explained by the fact that in the low-threshold range, more symbols with incorrect detection will be forwarded to the destination and degrade the performance of the system. In the high-threshold range, fewer symbols will be forwarded to the destination, and the performance is influenced with the increase of the threshold. Next, we compare the BEP performance of the proposed scheme with other schemes: i) LLR-based threshold selective relaying scheme [19]; ii) LLR-based threshold selective relaying with ML detector [23]; iii) ideal relay selective scheme. In the simulations, the path loss factor is set as $\gamma=3$. Fig. 4 gives the simulation results of the four schemes.

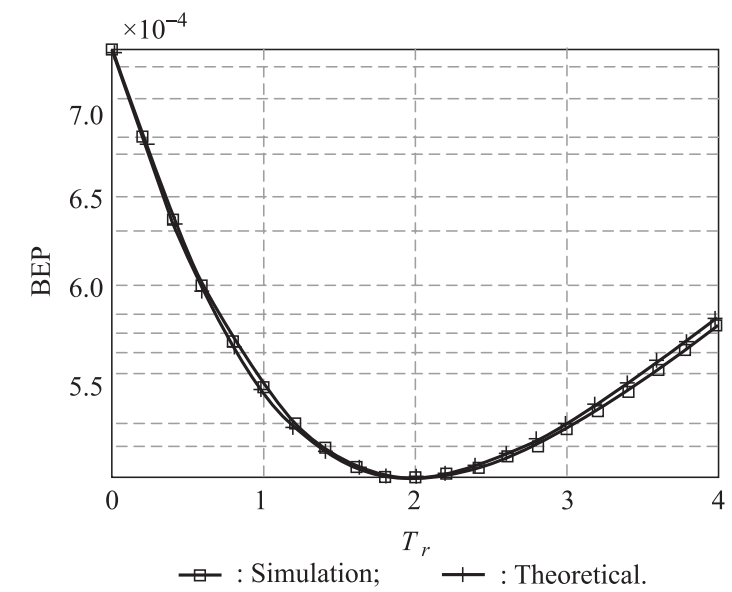

Fig. 3 Comparison of the theoretical BEP and the simulation BEP with respect to $T_{r}$ when $d_{s r}=1, d_{s r}=d_{r d}=1 / 2, \bar{\gamma}_{s d}=$ $\bar{\gamma}_{s r}=\bar{\gamma}_{r d}=15 \mathrm{~dB}$

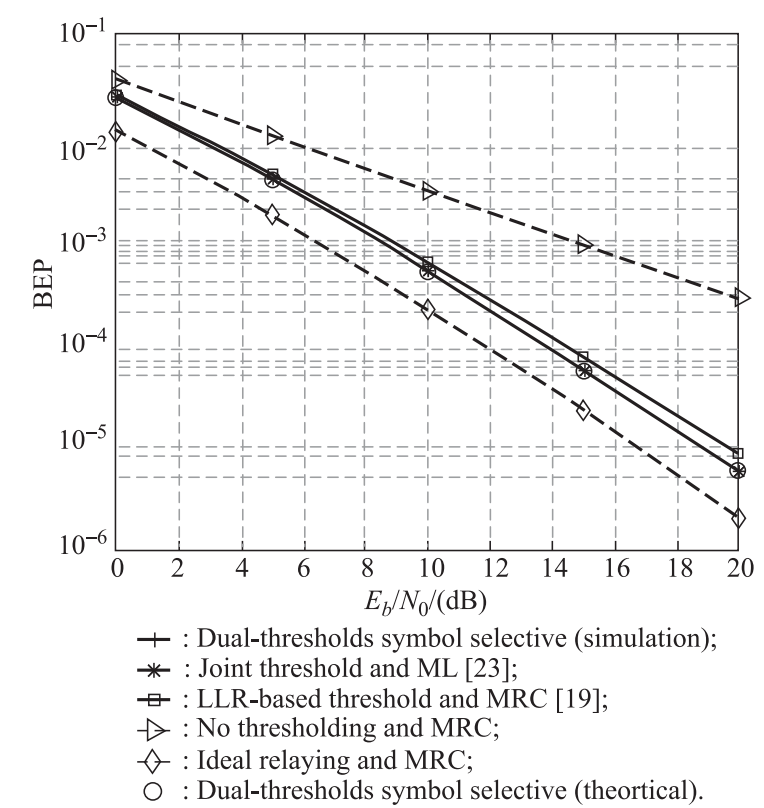

Fig. 4 E2E BEP performance comparisons when $d_{s d}=1, d_{s r}=$ $d_{r d}=1 / 2, \bar{\gamma}_{s d}=\bar{\gamma}_{s r}=\bar{\gamma}_{r d}$

In Fig. 4, the "no thresholding and MRC" represents the relay directly forwards symbols to the destination node. 
The "ideal relaying and MRC" indicates that the relay only forwards symbols that are correctly detected to the destination while discards symbols that are incorrect. Simulation results prove that the theoretical BEP of the proposed scheme closely matches the simulated ones. We observe that the performance of the proposed schemes is close to that of scheme [23], and both of them achieve a better performance than LLR-based threshold and MRC [19].

Further, we compare the computational complexity of the proposed method with the method in [23]. Table 2 shows the number of real multiplications and additions of the two methods with a frame length of $10^{7}$ bits. It can be observed from Table 2 that the numerical computation cost required by the proposed scheme is much less than the scheme [23], especially in high SNR. This accords with the analytical results in Table 1.

Table 2 Computation cost at the destination of the proposed scheme and scheme [23] when $L=10^{7}, d_{s d}=1, d_{s r}=d_{r d}=1 / 2$, $\bar{\gamma}_{s d}=\bar{\gamma}_{s r}=\bar{\gamma}_{r d}$

\begin{tabular}{cccc}
\hline \multirow{2}{*}{ SNR/dB } & \multirow{2}{*}{ Scheme } & \multicolumn{2}{c}{ Computational cost } \\
\cline { 3 - 4 } & & Multiplication & Addition \\
\hline \multirow{2}{*}{0} & Proposed scheme & 19405410 & 6440826 \\
& Scheme [23] & 19418974 & 75351792 \\
\hline \multirow{2}{*}{10} & Proposed scheme & 19912128 & 1897254 \\
& Scheme [23] & 19912059 & 79296472 \\
\hline \multirow{2}{*}{20} & Proposed scheme & 19990088 & 279889 \\
& Scheme [23] & 19990067 & 79920536 \\
\hline
\end{tabular}

\section{Conclusions}

In this paper, we propose an LLR-based dual-threshold symbol selective DmF scheme for three-node cooperative networks. The thresholds are used at the relay and the destination to measure the reliability of received signals, respectively. The relay only forwards reliable symbols to the destination to mitigate error propagation. At the destination, the computing burden is reduced by only combining unreliable symbols from the source with the symbols from the relay. To achieve the LLR-based thresholds, we derive E2E BEP under BPSK. Other than the scheme in [23], closed-form solutions of the thresholds are derived. Simulation results show that the analytical thresholds are close to the simulation ones. And it also proves that the computation costs of the proposed method is less than the method in [23] with almost the same BEP performance.

\section{References}

[1] J. N. Laneman, D. N. C. Tse, G. W. Wornell. Cooperative diversity in wireless networks: efficient protocols and outage behavior. IEEE Trans. on Information Theory, 2004, 50(12): $3062-3080$.

[2] Y. W. P. Hong, W. J. Huang, C. C. J. Kuo. Cooperative communications and networking. Technologies and system design. 1st ed. New York: Springer Science \& Business Media, 2010.
[3] J. N. Laneman. Modulation and demodulation for cooperative diversity in wireless systems. IEEE Trans. on Wireless Communications, 2006, 5(7): 1785-1794.

[4] K. S. Gomadam, S. A. Jafar. Optimizing soft information in relay networks. Proc. of the Asilomar Conference on Signals, Systems and Computers, 2006, 1(1): 18-22.

[5] K. S. Gomadam, S. A. Jafar. Optimal relay functionality for SNR maximization in memoryless relay networks. IEEE Journal on Selected Areas in Communications, 2007, 25(2): 390401.

[6] F. A. Onat, Y. Fan, H. Yanikomeroglu, et al. Threshold based relay selection in cooperative wireless networks. Proc. of IEEE Global Telecommunications Conference, 2008: 4810-4814.

[7] F. Onat, Y. Fan, H. Yanikomeroglu, et al. Asymptotic BER analysis of threshold digital relaying schemes in cooperative wireless systems. IEEE Trans. on Wireless Communications, 2008, 7(12): 4938 - 4947.

[8] F. A. Onat, A. Adinoyi, Y. Fan, et al. Threshold selection for SNR-based selective digital relaying in cooperative wireless networks. IEEE Trans. on Wireless Communications, 2008, 7(11): $4226-4237$.

[9] S. Ikki, M. H. Ahmed. Performance of decode-and-forward cooperative diversity networks over nakagami-m fading channels. Proc. of IEEE Global Telecommunications Conference, 2007: $4328-4333$.

[10] A. H. Bastami, A. Olfat. Optimal SNR-based selection relaying scheme in multi-relay cooperative networks with distributed space-time coding. IET Communications, 2010, 4(6): $619-630$.

[11] C. He, J. Liu, C. Zhai, et al. Performance analysis of SNRbased hybrid decode-amplify-forward cooperative diversity networks over Rayleigh fading channels. Proc. of IEEE Wireless Communications and Networking Conference, 2010: 16.

[12] S. Bouanen, H. Boujemaa, W. Ajib. Threshold-based adaptive decode-amplify-forward relaying protocol for cooperative systems. Proc. of the 7th International Wireless Communications and Mobile Computing Conference, 2011: 725-730.

[13] I. K. Sileh, W. Xiang, A. Maxwell. Error probability of OFDM-based hybrid relay protocols over wideband fading channels. Proc. of IEEE International Conference on InUltraWideband, 2013: 255-260.

[14] L. Daoud, A. El-Mahdy. Performance of hybrid relaying in cooperative wireless systems in the presence of impulsive noise. Signal Processing: Algorithms, Architectures, Arrangements, and Applications (SPA), 2014: 154-159.

[15] Z. Bai, J. Jia, C. X. Wang, et al. Performance analysis of SNR-based incremental hybrid decode-amplify-forward cooperative relaying protocol. IEEE Trans. on Communications, 2015, 63(6): 2094-2106.

[16] A. El-Mahdy, W. Alexan. A comparative study on the performance of LLR-and SNR-based hybrid relaying schemes. Wireless Communications and Mobile Computing, 2017, 2017(11): $1-7$.

[17] H. Van Khuong, H. Y. Kong. LLR-based decode-and-forward protocol for relay networks and closed-form BER expressions. IEICE Trans. on Fundamentals of Electronics, Communications and Computer Sciences, 2006, E89-A(6): 1832 - 1841.

[18] R. C. Palat, A. Annamalai, J. H. Reed. Log-likelihood-ratio based selective decode and forward cooperative communication. Proc. of IEEE Vehicular Technology Conference, 2008, 1: $615-618$.

[19] T. Kwon, S. Lim, W. Seo, et al. LLR-based symbol selective transmission with a near-optimal threshold to minimize BEP for demodulation-forward relay systems. IEEE Trans. on Wire- 
less Communications, 2010, 9(2): 540-545.

[20] G. Al-Habian, A. Ghrayeb, M. Hasna, et al. Threshold-based relaying in coded cooperative networks. IEEE Trans. on Vehicular Technology, 2011, 60(1): 123-135.

[21] W. Alexan, A. El-Mahdy. A hybrid relaying protocol for wireless cooperative networks based on the log-likelihood ratio. Signal Processing: Algorithms, Architectures, Arrangements, and Applications (SPA), 2015: 128 - 133.

[22] S. S. Ikki, M. H. Ahmed. Performance analysis of incrementalrelaying cooperative-diversity networks over Rayleigh fading channels. IET Communications, 2011, 5(3): 337-349.

[23] X. N. Zeng, A. Ghrayeb, M. Hasna. Joint optimal thresholdbased relaying and ML detection in cooperative networks. IEEE Communation Letters, 2012, 16(6): 773 - 776.

[24] X. N. Zeng, A. Ghrayeb, M. Hasna. Joint optimal thresholdbased relaying and $\mathrm{ml}$ detection in network-coded two-way relay channels. IEEE Trans. on Communications, 2012, 60(9): $2657-2667$.

[25] A. Sendonaris, E. Erkip, B. Aazhang. User cooperation diversity-part II: implementation aspects and performance analysis. IEEE Trans. on Communications, 2003, 51(11): $1939-1948$.

[26] W. T. Wang, A. Cano, G. B. Giannakis. Efficient demodulation in cooperative schemes using decode-and-forward relays. Proc. of Conference Record of the 39th Asilomar Conference on Signals, Systems and Computers, 2005: 1051-1055.

[27] T. Wang, A. Cano, G. B. Giannakis, et al. High-performance cooperative demodulation with decode-and-forward relays. IEEE Trans. on Communications, 2007, 55(7): 1427-1438.

[28] D. G. Brennan. Linear diversity combining techniques. Proceedings of the IEEE, 2003, 91(2): $331-356$.

[29] J. G. Proakis. Digital communications. 5th Ed. New York: McGraw-Hill, 2007.

[30] Z. B. Zabinsky. Stochastic adaptive search for global optimization. New York: Springer, 2003.

\section{Biographies}

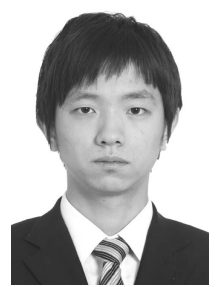

Peiyao Yang was born in 1989. He received his B.S. degree in information security and antagonism from Beijing Institute of Technology, Beijing, China, in 2011. Currently, he is pursuing his Ph.D. degree in the Professional Mobile Communication Research Group, School of Information and Electronics, Beijing Institute of Technology, China. His research interests include channel coding for wireless communications and cooperative communications. E-mail: yangpy@outlook.com

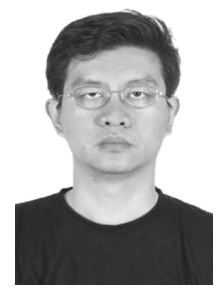

Hai Li was born in 1972. He received his B.S. and $\mathrm{Ph} . \mathrm{D}$. degrees in information and communication engineering from Beijing Institute of Technology in 1997 and 2002, respectively. He has co-authored over 30 papers published in scientific journals or conference proceedings. He is currently working at Beijing Institute of Technology as an associate professor. His current research interests include embedded system design and communication protocol engineering. E-mail: haili@bit.edu.cn

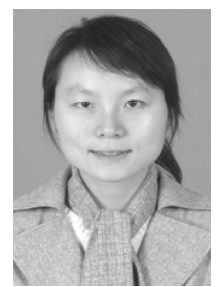

Shujuan Hou was born in 1977. She received her $\mathrm{Ph} . \mathrm{D}$. degree in signal and information processing from Beijing Institute of Technology in 2005. Currently, she is an associate professor of signal and information processing in Beijing Institute of Technology. Her main research interest is signal processing of mobile communication systems.

E-mail: shujuanhou@bit.edu.cn 